\title{
Detection of HPV DNA in paraffin- embedded cervical samples: a comparison of four genotyping methods
}

Felipe A. Castro ${ }^{1}$, Jill Koshiol' ${ }^{1}$ Wim Quint ${ }^{2}$, Cosette M. Wheeler ${ }^{4}$, Maura L. Gillison ${ }^{3}$, Laurence M. Vaughan ${ }^{5}$, Bernhard Kleter ${ }^{2}$, Leen-Jan van Doorn², Anil K. Chaturvedi ${ }^{1}$, Allan Hildesheim', Mark Schiffman ${ }^{1}$, Sophia S. Wang ${ }^{6}$, Rosemary E. Zuna ${ }^{7}$, Joan L. Walker ${ }^{7}$, S. Terence Dunn ${ }^{7}$ and Nicolas Wentzensen ${ }^{1,8^{*}}$

\begin{abstract}
Background: Identification of human papillomavirus (HPV) DNA in cervical tissue is important for understanding cervical carcinogenesis and for evaluating cervical cancer prevention approaches. However, HPV genotyping using formalin-fixed, paraffin-embedded (FFPE) tissues is technically challenging. We evaluated the performance of four commonly used genotyping methods on FFPE cervical specimens conducted in different laboratories and compared to genotyping results from cytological samples.

Methods: We included 60 pairs of exfoliated-cell and FFPE specimens from women with histologically confirmed cervical intraepithelial lesions grade 2 or 3. Cytology specimens were genotyped using the Linear Array assay. Four expert laboratories processed tissue specimens using different preparation methods and then genotyped the resultant sample preparations using four different HPV genotyping methods: SPF $_{10}-$ PCR DEIA LiPA 25 (version 1), Inno-LiPA, Linear Array and the Onclarity assay. Percentage agreement, kappa statistics and McNemar's chi-square were calculated for each comparison of different methods and specimen types.

Results: Overall agreement with respect to carcinogenic HPV status for FFPE samples between different methods was: 81.7, 86.7 and $91.7 \%$ for Onclarity versus Inno-LiPA, Linear Array and SPF-LiPA 25 , respectively; 81.7 and $85.0 \%$ for Linear Array versus Inno-LiPA and SPF-LiPA 25 , respectively; and $86.7 \%$ for SPF-LiPA 25 versus Inno-LiPA.

Type-specific agreement was $>88.3 \%$ for all pair-wise comparisons. Comparisons with cytology specimens resulted in overall agreements from 80 to $95 \%$ depending on the method and type-specific agreement was $>90 \%$ for most comparisons.
\end{abstract}

Conclusions: Our data demonstrate that the four genotyping methods run by expert laboratories reliably detect HPV DNA in FFPE specimens with some variation in genotype-specific detection.

Keywords: Genotyping, Formalin-fixed paraffin-embedded tissues, Cervical intraepithelial lesions, Exfoliated-cell

\section{Background}

The causal role of HPV in cervical carcinoma has been well established. Persistent infection with one of 13-15 oncogenic types of human papillomavirus (HPV) is a necessary cause of cervical cancer [1,2]. Diagnosis of HPV infection is usually performed from cervical cytology

\footnotetext{
* Correspondence: wentzenn@mail.nih.gov

'Division of Cancer Epidemiology and Genetics, National Cancer Institute, Bethesda, MD, USA

${ }^{8}$ Division of Cancer Epidemiology \& Genetics, NCI Shady Grove, 9609 Medical Center Drive 7E114, Rockville, MD 20850, USA

Full list of author information is available at the end of the article
}

specimens and used in natural history studies, implementation of screening programs, and follow-up of vaccination studies $[3,4]$. However, cytology specimens often contain larger numbers of HPV infections [5], most of which are thought to be transient infections, whereas it is assumed that a lesion is caused by one genotype [6, 7]. Thus, identification of HPV in tissue specimens is important to ascertain the causal type involved in HPV-related carcinogenesis.

Detection and classification of HPV infection through polymerase chain reaction (PCR)-based methods has 
been successfully implemented in cervical cytology specimens [8]. Genotyping methods for HPV vary by target sequence and amplicon size. Most assays target the L1 gene and some the viral oncogenes E6/E7. Amplicon sizes range from 65 base pairs (SPF10 primers) to 450 base pairs (PGMY09/11 primers). Standard genotyping methods for cytology specimens often cannot be easily applied to tissue specimens. Particularly, formalin fixation may cause extensive DNA damage, including cross-linking and fragmentation $[9,10]$. Consequently, it has been reported that successful amplification of HPV sequences from archival FFPE specimens is inversely correlated to the length of the amplicon of the PCR method and that specimen age may contribute to degradation $[11,12]$. Furthermore, differences in sample processing and DNA extraction of FFPE materials may explain discrepancies observed in the performance of specific genotyping methods for FFPE specimens [13-15]. Several recent reports suggest that the use of robust extraction methods can improve the performance of nucleic acid tests when applied to fixed specimen types [16-18].

Given the importance of accurately identifying HPV present in FFPE materials and the lack of studies comparing HPV assays in tissue specimens, we evaluated HPV genotyping methods in FFPE specimens using four HPV genotyping methods, representing frequently-used assays with well-documented performance in cervical specimens. The laboratories participating in this comparison have long-standing experience in HPV genotyping and they have participated in many inter-laboratory comparisons, including WHO HPV LabNet proficiency testing.

\section{Methods}

\section{Study population}

The materials for this study were obtained from the Study to Understand Cervical Cancer Early Endpoints and Determinants (SUCCEED), a cross-sectional study including women referred to the University of Oklahoma from November 2003 to September 2009 for abnormal cervical screening results. SUCCEED design and methodology, including the details on enrollment, questionnaire data, HPV DNA genotyping, histology, and cytology procedures, have been described in depth elsewhere [19, 20]. Participants (median age 30.0 years) signed informed consent, completed interviewer-administered, standardized questionnaires and provided liquid-based cytology specimens for ThinPrep Pap and HPV genotyping. According to management guidelines, most histologically confirmed high-grade lesions diagnosed as cervical intraepithelial neoplasia grade 2 (CIN2) and all CIN3 were treated by loop electrosurgical excision procedure (LEEP) of the transformation zone.

For the present study, we selected 60 women diagnosed with CIN2+ (29 CIN2 and 31 CIN3) for whom tissue blocks were available and who had cytology-based HPV DNA genotyping results. The median duration between cytology sampling and LEEP was 27.5 days. In 26 women, cytology and LEEP were done on the same day.

Institutional Review Board approval for this study was provided by University of Oklahoma and the U.S. National Cancer Institute.

\section{HPV genotyping of cytology specimens}

HPV detection and genotyping in cytology specimens was done using the Linear Array HPV Genotyping Test (Roche Molecular Diagnostics), as previously described $[19,20]$. Briefly, the Linear Array assay is a type-specific, PGMY09/11 L1 primer PCR-based assay for $37 \mathrm{HPV}$ types, 13 high- and 24 low-risk HPV types $(6,11,16,18$, $26,31,33,35,39,40,42,45,51,52,53,54,55,56,58$, $59,61,62,64,66,67,68,69,70,71,72,73,81,82,83$, 84, and 89). HPV 52 is not determined directly by a type-specific probe but rather by a probe that cross hybridizes with HPV 33, 35, 52, and 58. The presence of HPV 52 was inferred only if the cross-reactive probe was hybridized but there was no hybridization detected for the HPV 33, 35 and 58 type-specific probes. Notably, concurrent infections of type 52 with the three other types cannot be detected. The procedure followed recommendations of the manufacturer with the variations that DNA was isolated from $1 \mathrm{~mL}$ of exfoliated cells in PreservCyt using Qiagen DNeasy Blood and Tissue Kit, $10 \mu \mathrm{L}$ of template DNA was amplified, and the amplified products were hybridized and detected using an automated Auto-line probe assay (LiPA) staining system using $2.5 \mathrm{~mL}$ of each reagent per strip. The Linear Array results on the strips were evaluated by two independent observers. An unambiguous, continuous band was judged to indicate a positive result.

\section{Tissue sectioning}

A series of 4- $\mu$ m-thick tissue sections was cut from each paraffin block. The first and last sections were stained with hematoxylin and eosin (H\&E) to confirm the diagnosis of CIN2+. Each participating laboratory received two unstained 4um sections for HPV genotyping. Standard measures to avoid cross-contamination were taken during tissue sectioning and processing.

\section{HPV genotyping of tissue specimens}

One of four HPV genotyping methods was used to genotype tissue specimens in each specific laboratory: 1) the The BD Onclarity ${ }^{\text {max }}$ HPV Assay (denoted as "Onclarity"); 2) the Inno-LiPA system (denoted as "Inno-LiPA"); 3) the PGMY09/11 Linear Array (denoted as "Linear Array"); and 4) $\mathrm{SPF}_{10}$-DEIA and $\mathrm{LiPA}_{25}$ (denoted as "SPF$\mathrm{LiPA}_{25}$ "). The main features of each HPV method are listed in Table 1 and described below. All FFPE sections, 
Table 1 Formalin-fixed and paraffin embedded (FFPE) specimen processing, cytology specimens and human papillomavirus (HPV) testing methods

\begin{tabular}{|c|c|c|c|c|c|}
\hline \multirow[b]{2}{*}{$\underline{\text { Characteristics }}$} & \multirow{2}{*}{$\begin{array}{l}\text { Cytology specimens } \\
\text { Linear array }\end{array}$} & \multicolumn{4}{|c|}{ HPV genotyping methods/protocol for FFPE specimens } \\
\hline & & Onclarity & Inno-LiPA & Linear array & SPF-LiPA 25 \\
\hline Deparaffinization & NA & No & Yes & No & No \\
\hline DNA extraction & Crude extraction & $\begin{array}{l}\text { One-step heat / chemical } \\
\text { lysis + FOX }{ }^{\mathrm{TM}} \text { magnetic } \\
\text { particle DNA purification }\end{array}$ & $\begin{array}{l}\text { Xylene, proteinase K, } \\
\text { phenol-chloroform extraction, } \\
\text { ethanol precipitation }\end{array}$ & Crude extraction & Crude extraction \\
\hline HPV testing & PGMY09/11 Linear Array & $\begin{array}{l}\text { The BD Onclarity }{ }^{\mathrm{TM}} \\
\text { HPV Assay }\end{array}$ & Inno-LiPA & $\begin{array}{l}\text { PGMY09/11 } \\
\text { Linear Array }\end{array}$ & $\begin{array}{l}\mathrm{SPF}_{10}-\mathrm{DEIA}, \mathrm{LiPA}_{25} \\
\text { (version 1) }\end{array}$ \\
\hline $\begin{array}{l}\text { Probes for } \\
\text { oncogenic } \\
\text { types detectable }\end{array}$ & $\begin{array}{l}16,18,31,33,35,39,45,51 \\
56,58,59,66,68 \text { and } 52 / \\
33 / 35 / 58\end{array}$ & $\begin{array}{l}16,18,31,45,51,52,33 / \\
58,39 / 68 / 35, \text { and } 59 / \\
56 / 66\end{array}$ & $\begin{array}{l}16,18,31,33,35,39,45 \\
51,52,56,58,59,66 \\
\text { and } 68\end{array}$ & $\begin{array}{l}16,18,31,33,35,39 \\
45,51,56,58,59,66 \\
68, \text { and } 52 / 33 / 35 / 58\end{array}$ & $\begin{array}{l}16,18,31,33,35,39 \\
45,51,52,56,58,59 \\
66 \text { and } 68 / 73\end{array}$ \\
\hline $\begin{array}{l}\text { Amplicon } \\
\text { length (bp) }\end{array}$ & 450 & 79-137 & 65 & 450 & 65 \\
\hline
\end{tabular}

Onclarity, The BD Onclarity ${ }^{\mathrm{TM}}$ HPV Assay; Inno-LiPA, The Inno-LiPA system; Linear Array, The PGMY09/11 Linear Array, and SPF-LiPA 25 , The SPF 10 -DEIA, LiPA 25 (version 1) NA, not applicable; HPV 52 is positively identified in the linear Array when the presence of HPV 33, 35, and 58 is excluded

including those that tested HPV-negative, were positive for human DNA controls

\section{Onclarity assay}

The BD Onclarity ${ }^{\text {tx }}$ HPV Assay (BD Diagnostics, Sparks, USA) is a real-time PCR assay that detects type-specific $E 6$ and E7 genomic DNA. It simultaneously detects all 14 high-risk HPV types, and can provide genotyping information on six individual genotypes (HPV 16, 18, 31, 45, 51 and 52), reporting the remaining HPV types in three distinct groups (33 and 58; 56, 59 and 66; and 35, 39 and 68). Each FFPE patient sample was extracted using the automated workflow on the Viper ${ }^{\mathrm{Tm}}$ LT system. The section was combined with $0.5 \mathrm{~mL}$ of distilled water and added directly to a tube with pierceable cap containing a proprietary diluent. The sample was then lysed directly using the Viper ${ }^{\mathrm{rw}}$ LT Pre-warm station before being transferred onto the deck of the instrument where it underwent automated sample processing and PCR detection. Briefly, the DNA was extracted using BD FOX ${ }^{\mathrm{mm}}$ magnetic particles and the eluate-containing DNA was used to set up three PCR genotyping reactions: G1 detects HPV 16, HPV 18 and HPV 45 plus the internal beta globin control; G2 detects HPV 31, HPV 33_58 and HPV 56_59_66 plus the internal beta globin control; G3 detects HPV 51, HPV 52 and HPV 35_39_68 plus the internal beta globin control. After 40 PCR cycles, any Ct score for a specific genotype and/or the internal beta globin control was considered positive for that channel.

\section{Inno-LiPA assay}

DNA was purified from paraffin-embedded material as previously described [21]. Purified DNA was evaluated for the presence of HPV DNA by use of the Inno-LiPA assay (Innogenetics, Gent, Belgium), an assay that utilizes the $\mathrm{SPF}_{10}$ consensus primer system to amplify a $65 \mathrm{bp}$ fragment of the L1 region of HPV, followed by reverse line blot hybridization to HPV type-specific immobilized probes for 18 high-risk/ possibly high-risk $(16,18,25,31,33,35,39,45,51,52,53,56,58,59,66$, $68,73,82)$ and 7 low-risk $(6,11,40,43,44,54,70) \mathrm{HPV}$ types. In addition to the internal assay control, testing for human DNA was conducted from all specimens (ERV3). The line probe assays are evaluated by two independent observers and were adjudicated by a third observer when different results are reported, which did not occur in this study.

\section{Linear array assay}

Without removal of paraffin wax, the tissue sections obtained for HPV genotyping were resuspended (50-125 $\mu \mathrm{l})$ in $10 \mathrm{mM}$ Tris $\mathrm{pH} 8.0$ containing $1 \mathrm{~mm}$ EDTA, $0.1 \%$ Laureth-12 and $1 \mathrm{mg} / \mathrm{ml}$ proteinase $\mathrm{K}$ (PK) and digested with shaking at $65^{\circ} \mathrm{C}$ for $4 \mathrm{~h}$ followed by overnight at $37^{\circ}$ C. Prior to polymerase chain reaction (PCR)-based HPV genotyping, PK was inactivated at $95{ }^{\circ} \mathrm{C}$ for $15 \mathrm{~min}$. Microfuge tubes were immediately centrifuged briefly at $13,000 \mathrm{X} g$ while the paraffin-wax was liquefied and an aqueous-wax interface formed upon cooling. Two and five microliters of the aqueous digest from each tissue specimen were used for genotyping with the LINEAR ARRAY HPV Genotyping Test (HPV LA; Roche Diagnostics, Indianapolis, Indiana USA). The LA HPV Genotyping Test is a qualitative test for 37 HPV genotypes incorporating selective PCR amplification with biotinylated PGMY 09/11 L1 region consensus primers and colorimetric detection of amplified products bound to immobilized HPV genotype -related oligonucleotide probes on a LINEAR ARRAY HPV genotyping strip. PGMY-based HPV genotyping with the HPV LA have been previously reported in detail [22, 23]. Using the Roche HPV LA detection kit, hybridizations were automated using Tecan ProfiBlot-48 robots (Tecan, Austria) as previously described [24]. Two independent readers interpreted the presence of HPV 
genotypes using a reference template provided by the manufacturer. Any discrepancies identified between the two readers were adjudicated by a third review.

\section{SPF-LiPA 25 assay}

Total DNA was isolated from FFPE tissue material by proteinase $\mathrm{K}$ treatment, $250 \mu \mathrm{l}$ proteinase $\mathrm{K}$ lysis buffer was added and incubated at $56{ }^{\circ} \mathrm{C}$ for $16-24$ h. Proteinase $\mathrm{K}$ was heat-inactivated by incubation at $96{ }^{\circ} \mathrm{C}$ for 10 min. Each DNA isolation run and PCR run contained HPV positive and negative controls.

Specimens were tested for HPV DNA by PCR amplification/typing using the HPV SPF 10 PCR DEIA and LiPA $_{25}$ version 1 assay (Labo Biomedical Products, Rijswijk, The Netherlands) based on licensed Innogenetics technology $[25,26]$. The $\mathrm{SPF}_{10}$ PCR primer set detects a broad spectrum of HPV genotypes by amplification of a small fragment of $65 \mathrm{bp}$ from the $L 1$ region of HPV. Reverse primers contain a biotin label at the 5 ' end, enabling capture of the reverse strand onto streptavidincoated microtiter plates. Captured amplimers are then denatured by alkaline treatment, and detected by a defined cocktail of digoxigenin-labeled probes, allowing detection of a broad spectrum of HPV genotypes. This method is designated an HPV DNA enzyme immunoassay (DEIA), and the results are an optical density value. When a sample is HPV positive by DEIA, the same $\mathrm{SPF}_{10}$ amplimer is also used to identify the HPV genotype by reverse hybridization to the $\mathrm{LiPA}_{25}$ genotyping strip (version 1). This line probe assay contains probes for 25 different HPV genotypes (i.e., HPV types $6,11,16,18,31,33,34,35,39,40,42,43,44,45,51,52$, $53,54,56,58,59,66,68 / 73,70$, and 74$)$. The test results, purple lines on a LiPA strip are visually scored by two independent readers. In case of a discrepant line score a third reader determines the final outcome. In this study there were no different scores.

In SPF10 DEIA positive samples the presence of HPV 16 and 18 was also tested by type-specific (TS) PCR primer sets. TS16 and TS18 amplimers were detected by DEIA, similar to the method for SPF10 amplimer detection. The final genotyping result is the outcome of the testing algorithm [27]. As a result of this algorithm HPV16 and HPV 18 were only detected by type specific PCR three times and one time, respectively. These types were found in the presence of another HPV type. The SPF10 LiPA assay does not use an internal human DNA control. Two specimens were negative for all HPV types and 10fold dilution with and without spiking using HPV16 as a target did not indicate PCR inhibition.

\section{Statistical analyses}

Analyses were limited to $14 \mathrm{HPV}$ types classified as carcinogenic $(16,18,31,33,35,39,45,51,52,56,58,59$,
66, 68) [2] and samples positive for other HPV types were considered as negative for HPV. We calculated overall and type-specific prevalence for high-risk HPV types detected in exfoliated cells by Linear Array and in FFPE specimens across all different methods.

To evaluate the performance of each method in FFPE tissue samples, we compared FFPE HPV genotyping results between different HPV methods. However, pairwise comparisons were restricted to those carcinogenic types for which a specific probe was available across all of the different methods. Thus, comparison across all four assays was possible for types 16, 18, 31, 45, 51 and 52, and across 3 assays (Inno-LiPA, Linear Array and SPF-LiPA 25 ) for types 33, 35, 39, 56 and 58, and 66. HPV type 68 genotyping was only compared between Linear Array and Inno-LiPA. HPV genotyping concordance was tested by calculating overall and type-specific percentages of agreement and kappa values. McNemar's chi-square was calculated to test for significance $(\mathrm{p}<0.05)$ in the pattern of disagreement.

An indirect analysis that may allow a further evaluation of the performance of these four genotyping methods in FFPE samples is compare FFPE genotyping results with those of their paired cytological sample. HPV genotyping results from FFPE specimens for each of the four assays was compared to results from cytology specimens as described above, and by creating three categories of agreement: (i) Identical: when the same number and type of HPV was identified in both specimens; (ii) Compatible: when at least one HPV type was found in common; and (iii) Discrepant: when no type was identified in common or the FFPE sample was HPV negative. This classification was performed, first by including all single and combined HPV type probes available in each of the assays and second, by including only single probes.

\section{Results}

\section{Overall and type-specific HPV prevalence}

All 60 exfoliated cell specimens were positive for at least one of the 14 carcinogenic HPV genotypes tested by Linear Array (Table 2). The most prevalent types were HPV16 (53.3 \%), 18 (15.0\%), 31 and 45 (both $13.3 \%$ ). Multiple HPV types were found in $35 \%$ of the exfoliated cell specimens.

Eighty percent or more of the FFPE specimens were positive for at least one HPV type in each of the four methods (Table 2). Type-specific positivity ranged across different HPV methods from 0 \% (for HPV 35, 59, 66, and 68 in some of the tested methods) to $48.3 \%$ (for HPV 16 using the SPF-LiPA 25 method). The SPF-LiPA 25 assay identified more types at both the overall and the type-specific level. Detection of multiple types was rare among FFPE specimens by all methods (from $5.0 \%$ in 
Table 2 Human papillomavirus (HPV) genotyping results for paired cytology and tissue specimens ${ }^{a}$ by genotyping method (\%)

\begin{tabular}{|c|c|c|c|c|c|}
\hline \multirow[b]{2}{*}{ HPV, type } & \multirow{2}{*}{$\begin{array}{l}\text { Cytology } \\
\text { Linear Array }\end{array}$} & \multicolumn{4}{|c|}{ FFPE samples } \\
\hline & & Onclarity & Inno-LiPA & Linear Array & SPF-LiPA 25 \\
\hline Any oncogenic ${ }^{b}$ & $60(100.0)$ & $54(90.0)$ & $51(85.0)$ & $48(80.0)$ & $57(95.0)$ \\
\hline \multicolumn{6}{|l|}{ Single probes } \\
\hline HPV 16 & $32(53.3)$ & $26(43.3)$ & $25(41.7)$ & $24(40.0)$ & $29(48.3)$ \\
\hline HPV 18 & $9(15.0)$ & $8(13.3)$ & $7(11.7)$ & $5(8.3)$ & $8(13.3)$ \\
\hline HPV 31 & $8(13.3)$ & $7(11.7)$ & $7(11.7)$ & $6(10.0)$ & $7(11.7)$ \\
\hline HPV 33 & $2(3.3)$ & NA & $2(3.3)$ & $2(3.3)$ & $2(3.3)$ \\
\hline HPV 35 & $1(1.7)$ & NA & 0 & 0 & 0 \\
\hline HPV 39 & $4(6.7)$ & NA & $2(3.3)$ & $2(3.3)$ & $2(3.3)$ \\
\hline HPV 45 & $8(13.3)$ & $5(8.3)$ & $2(3.3)$ & $3(5.0)$ & $5(8.3)$ \\
\hline HPV 51 & $6(10.0)$ & $1(1.7)$ & $2(3.3)$ & $1(1.7)$ & $2(3.3)$ \\
\hline HPV 52 & NA & $5(8.3)$ & $3(5.0)$ & NA & $6(10.0)$ \\
\hline HPV 56 & $4(6.7)$ & NA & $1(1.7)$ & $1(1.7)$ & $1(1.7)$ \\
\hline HPV 58 & $5(8.3)$ & NA & $3(5.0)$ & $3(5.0)$ & $3(5.0)$ \\
\hline HPV 59 & $2(3.3)$ & NA & 0 & $2(3.3)$ & $1(1.7)$ \\
\hline HPV 66 & $4(6.7)$ & NA & 0 & 0 & $1(1.7)$ \\
\hline HPV 68 & $2(3.3)$ & NA & 0 & $1(1.7)$ & NA \\
\hline \multicolumn{6}{|l|}{ Combined probes } \\
\hline $52 / 33 / 35 / 58^{c}$ & $6(10.0)$ & NA & NA & $2(3.3)$ & NA \\
\hline $33 / 58$ & NA & $5(8.3)$ & NA & NA & NA \\
\hline $35 / 39 / 68$ & NA & $3(5.0)$ & NA & NA & NA \\
\hline $56 / 59 / 66$ & NA & $4(6.7)$ & NA & NA & NA \\
\hline $68 / 73$ & NA & NA & NA & NA & $2(3.3)$ \\
\hline Multiple infections ${ }^{d}$ & $21(35.0)$ & $10(16.6)$ & $3(5.0)$ & $4(6.7)$ & $11(18.3)$ \\
\hline
\end{tabular}

the Inno-LiPA to $18.3 \%$ in the SPF-LiPA 25 ). There was a notable difference in the detection rate of multiple types between tests with the Onclarity and SPF-LiPA 25 method detecting 2-3 times more multiple types than those detected by the Inno-LiPA and Linear Array methods (Table 2). Genotyping results for each of the participants are provided in Additional file 1: Table S1. Only one specimen was negative across all methods. In three cases, Onclarity detected types that were not found with any of the other assays. Six specimens were repeated in three labs; only one repeat results showed a partial discrepancy (Additional file 2: Table S2).

Multiple HPV genotypes were detected in 21 of the 60 exfoliated cell specimens. In the corresponding FFPE specimen multiple genotypes were only detected in eight specimens by at least one of the four different genotyping assays. This observation can be explained by the fact that exfoliated cell specimens cover the complete cervix area, whereas FFPE specimens are taken at a specific site of the cervix epithelium.

\section{Agreement between tissue-based HPV testing across HPV methods}

Overall concordance between tissue-based HPV results across laboratory methods was good (Table 3); the overall percent agreement ranged from 81.7 for Inno-LiPA compared with Linear Array and with Onclarity to $91.7 \%$ for SPF-LiPA 25 compared with Onclarity. The number of samples with HPV types detected was lower when samples were genotyped by Linear Array compared with the number of positive samples obtained by SPF-LiPA 25 (McNemar's p-value $=0.004)$. Percentage of agreement for the two most common HPV types, 16 and 18, ranged from $88.3 \%$ (SPF-LiPA 25 vs. Onclarity) to $95 \%$ (Onclarity vs. Linear Array) for HPV 16 and from 95 \% (SPF-LiPA 25 vs. Linear Array and Onclarity vs. Linear Array) to $98.3 \%$ (Onclarity vs. Inno-LiPA and SPF-LiPA 25 vs. Inno-LiPA) for HPV 18, but none of the differences were statistically significant. Summarized across all types, the percent identical, compatible and discrepant results for each assay combination is shown in Additional file 3: Table S3. 
Table 3 Human papillomavirus (HPV) genotyping agreement for tissue specimens* tested with four different genotyping methods

\begin{tabular}{|c|c|c|c|c|c|c|c|c|c|}
\hline \multirow{2}{*}{$\begin{array}{l}\text { HPV } \\
\text { Type }\end{array}$} & \multicolumn{2}{|l|}{ Method 1} & \multicolumn{2}{|l|}{ Method 2} & \multirow{2}{*}{$\begin{array}{l}\text { Percent }^{a} \\
\text { agreement }\end{array}$} & \multicolumn{3}{|l|}{ Kappa } & \multirow[b]{2}{*}{ P } \\
\hline & Name & $\overline{\text { Positive }}$ & Name & Positive & & Value & $95 \% \mathrm{Cl}$ & & \\
\hline \multicolumn{10}{|c|}{ Oncogenic $^{\mathrm{b}}$} \\
\hline Any & Onclarity & 54 & Inno-LiPA & 51 & 81.7 & 0.2 & -0.15 & 0.48 & 0.5 \\
\hline Any & Onclarity & 54 & Linear Array & 48 & 86.7 & 0.5 & 0.19 & 0.78 & 0.07 \\
\hline Any & Onclarity & 54 & SPF-LiPA 25 & 57 & 91.7 & 0.4 & -0.01 & 0.82 & 0.4 \\
\hline Any & Linear Array & 48 & Inno-LiPA & 51 & 81.7 & 0.4 & 0.07 & 0.67 & 0.5 \\
\hline Any & Linear Array & 48 & SPF-LiPA 25 & 57 & 85.0 & 0.3 & 0.05 & 0.65 & 0.004 \\
\hline Any & SPF-LiPA 25 & 57 & Inno-LiPA & 51 & 86.7 & 0.3 & -0.06 & 0.62 & 0.07 \\
\hline \multicolumn{10}{|c|}{ By single probe } \\
\hline 16 & Onclarity & 26 & Linear Array & 24 & 90.0 & 0.79 & 0.64 & 0.95 & 0.7 \\
\hline 16 & Onclarity & 26 & Inno-LiPA & 25 & 91.7 & 0.83 & 0.69 & 0.97 & 1.0 \\
\hline 16 & Linear Array & 24 & Inno-LiPA & 25 & 95.0 & 0.9 & 0.78 & 1.0 & 1.0 \\
\hline 16 & SPF-LiPA 25 & 29 & Onclarity & 26 & 88.3 & 0.77 & 0.6 & 0.93 & 0.5 \\
\hline 16 & SPF-LiPA 25 & 29 & Inno-LiPA & 25 & 90.0 & 0.8 & 0.65 & 0.95 & 0.2 \\
\hline 16 & SPF-LiPA 25 & 29 & Linear Array & 24 & 91.7 & 0.83 & 0.69 & 0.97 & 0.1 \\
\hline 18 & Onclarity & 8 & Inno-LiPA & 7 & 98.3 & 0.92 & 0.78 & 1.0 & 1.0 \\
\hline 18 & Onclarity & 8 & Linear Array & 5 & 95.0 & 0.74 & 0.47 & 1.0 & 0.3 \\
\hline 18 & Linear Array & 5 & Inno-LiPA & 7 & 96.7 & 0.82 & 0.57 & 1.0 & 0.5 \\
\hline 18 & SPF-LiPA 25 & 8 & Linear Array & 5 & 95.0 & 0.74 & 0.47 & 1.0 & 0.3 \\
\hline 18 & SPF-LiPA 25 & 8 & Inno-LiPA & 7 & 98.3 & 0.92 & 0.78 & 1.0 & 1.0 \\
\hline 18 & SPF-LiPA 25 & 8 & Onclarity & 8 & 96.7 & 0.86 & 0.66 & 1.0 & 1.0 \\
\hline 31 & Onclarity & 7 & Inno-LiPA & 7 & 96.7 & 0.84 & 0.62 & 1.0 & 1.0 \\
\hline 31 & Onclarity & 7 & Linear Array & 6 & 98.3 & 0.91 & 0.75 & 1.0 & 1.0 \\
\hline 31 & Linear Array & 6 & Inno-LiPA & 7 & 98.3 & 0.91 & 0.75 & 1.0 & 1.0 \\
\hline 31 & SPF-LiPA 25 & 7 & Linear Array & 6 & 98.3 & 0.91 & 0.75 & 1.0 & 1.0 \\
\hline 31 & SPF-LiPA 25 & 7 & Onclarity & 7 & 96.7 & 0.84 & 0.62 & 1.0 & 1.0 \\
\hline 31 & SPF-LiPA 25 & 7 & Inno-LiPA & 7 & 100 & NA & NA & NA & NA \\
\hline 33 & Linear Array & 2 & Inno-LiPA & 2 & 100 & NA & NA & NA & NA \\
\hline 33 & SPF-LiPA 25 & 2 & Linear Array & 2 & 100 & NA & NA & NA & NA \\
\hline 33 & SPF-LiPA 25 & 2 & Inno-LiPA & 2 & 100 & NA & NA & NA & NA \\
\hline 35 & Linear Array & 0 & Inno-LiPA & 0 & NA & NA & NA & NA & NA \\
\hline 35 & SPF-LiPA 25 & 0 & Inno-LiPA & 0 & NA & NA & NA & NA & NA \\
\hline 35 & SPF-LiPA 25 & 0 & Linear Array & 0 & NA & NA & NA & NA & NA \\
\hline 39 & Linear Array & 2 & Inno-LiPA & 2 & 100 & NA & NA & NA & NA \\
\hline 39 & SPF-LiPA 25 & 2 & Inno-LiPA & 2 & 100 & NA & NA & NA & NA \\
\hline 39 & SPF-LiPA 25 & 2 & Linear Array & 2 & 100 & NA & NA & NA & NA \\
\hline 45 & Onclarity & 5 & Inno-LiPA & 2 & 91.7 & 0.25 & -0.19 & 0.69 & 0.4 \\
\hline 45 & Onclarity & 5 & Linear Array & 3 & 93.3 & 0.47 & 0.03 & 0.91 & 0.6 \\
\hline 45 & Linear Array & 3 & Inno-LiPA & 2 & 95.0 & 0.38 & -0.18 & 0.93 & 1.0 \\
\hline 45 & SPF-LiPA 25 & 5 & Linear Array & 3 & 96.7 & 0.73 & 0.38 & 1.0 & 0.5 \\
\hline 45 & SPF-LiPA 25 & 5 & Inno-LiPA & 2 & 95.0 & 0.55 & 0.11 & 0.99 & 0.3 \\
\hline 45 & SPF-LiPA 25 & 5 & Onclarity & 5 & 96.7 & 0.78 & 0.49 & 1.0 & 1.0 \\
\hline 51 & Onclarity & 1 & Inno-LiPA & 2 & 98.3 & 0.66 & 0.04 & 1.0 & 1.0 \\
\hline 51 & Onclarity & 1 & Linear Array & 1 & 100 & NA & NA & NA & NA \\
\hline
\end{tabular}


Table 3 Human papillomavirus (HPV) genotyping agreement for tissue specimens* tested with four different genotyping methods (Continued)

\begin{tabular}{|c|c|c|c|c|c|c|c|c|c|}
\hline 51 & Linear Array & 1 & Inno-LiPA & 2 & 98.3 & 0.66 & 0.04 & 1.0 & 1.0 \\
\hline 51 & SPF-LiPA 25 & 2 & Linear Array & 1 & 98.3 & 0.66 & 0.04 & 1.0 & 1.0 \\
\hline 51 & SPF-LiPA 25 & 2 & Onclarity & 1 & 98.3 & 0.66 & 0.04 & 1.0 & 1.0 \\
\hline 51 & SPF-LiPA 25 & 2 & Inno-LiPA & 2 & 100 & NA & NA & NA & NA \\
\hline 52 & Onclarity & 5 & Inno-LiPA & 3 & 93.3 & 0.47 & 0.03 & 0.91 & 0.6 \\
\hline 52 & Onclarity & 5 & Linear Array & 2 & 95.0 & 0.55 & 0.11 & 0.99 & 0.3 \\
\hline 52 & Linear Array & 2 & Inno-LiPA & 3 & 98.3 & 0.79 & 0.4 & 1.0 & 1.0 \\
\hline 52 & SPF-LiPA 25 & 6 & Linear Array & 2 & 93.3 & 0.47 & 0.05 & 0.9 & 0.1 \\
\hline 52 & SPF-LiPA 25 & 6 & Inno-LiPA & 3 & 95.0 & 0.64 & 0.27 & 1.0 & 0.3 \\
\hline 52 & SPF-LiPA 25 & 6 & Onclarity & 5 & 95.0 & 0.7 & 0.38 & 1.0 & 1.0 \\
\hline 56 & Linear Array & 1 & Inno-LiPA & 1 & 100 & NA & NA & NA & NA \\
\hline 56 & SPF-LiPA 25 & 1 & Linear Array & 1 & 100 & NA & NA & NA & NA \\
\hline 56 & SPF-LiPA 25 & 1 & Inno-LiPA & 1 & 100 & NA & NA & NA & NA \\
\hline 58 & Linear Array & 3 & Inno-LiPA & 3 & 100 & NA & NA & NA & NA \\
\hline 58 & SPF-LiPA 25 & 3 & Linear Array & 3 & 100 & NA & NA & NA & NA \\
\hline 58 & SPF-LiPA 25 & 3 & Inno-LiPA & 3 & 100 & NA & NA & NA & NA \\
\hline 59 & Linear Array & 2 & Inno-LiPA & 0 & NA & NA & NA & NA & NA \\
\hline 59 & SPF-LiPA 25 & 1 & Linear Array & 2 & 98.3 & 0.66 & 0.04 & 1.0 & 1.0 \\
\hline 59 & SPF-LiPA 25 & 1 & Inno-LiPA & 0 & NA & NA & NA & NA & NA \\
\hline 66 & Linear Array & 0 & Inno-LiPA & 0 & NA & NA & NA & NA & NA \\
\hline 66 & SPF-LiPA 25 & 1 & Inno-LiPA & 0 & NA & NA & NA & NA & NA \\
\hline 66 & SPF-LiPA 25 & 1 & Linear Array & 0 & NA & NA & NA & NA & NA \\
\hline 68 & Linear Array & 1 & Inno-LiPA & 0 & NA & NA & NA & NA & NA \\
\hline
\end{tabular}

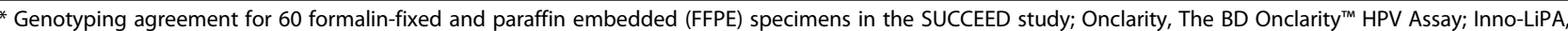
The Inno-LiPA system; Linear Array, The PGMY09/11 Linear Array, and SPF-LiPA 25 , The SPF $10-$ DEIA, LiPA $_{25}$ (version 1); $p, p$-value of the McNerman test; ${ }^{b}$ Any,

includes a specimen positive for any of the $14 \mathrm{HPV}$ types; NA, not apply or cannot be calculated; HPV 52 was positive when there was a test negative for HPV 33 ,

35 , and 58 individually but positive for the combined probe 52/33/35/58 (for Linear Array)

Agreement between exfoliated-cell- and tissue-based HPV testing

Identical HPV results between cytology and FFPE were found in $53.3 \%$ (Onclarity), $56.7 \%$ (Inno-LiPA), $53.3 \%$ (Linear Array), and $61.7 \%\left(\mathrm{SPF}-\mathrm{LiPA}_{25}\right)$ of the cases (Table 4); an additional 25.0 to $33.3 \%$ were categorized as compatible HPV results and 5.0 to $21.7 \%$ as discrepant results, meaning no type agreement or sample is HPV negative. Almost all discrepant results were related to a negative test result.

The overall agreement of HPV status between exfoliatedcell and FFPE specimens across the four tested methods was $90 \%$ for Onclarity, $85.0 \%$ for Inno-LiPA, $80 \%$ for Linear Array, and $95 \%$ for SPF-LiPA 25 (Table 4). When considering individual types, the percentage agreement ranged from $83.3 \%$ (HPV16) to 98.3 (HPV52) for the Onclarity method, $88.3 \%$ (HPV16) to $100 \%$ (HPV33) for the SPF-LiPA 25 and the Inno-LiPA methods, and from $86.7 \%$ (HPV 16) to $100 \%$ (HPV33 and 59) for the Linear Array method. Detection for HPV16 and 45 tested for the Inno-LiPA method and for HPV16 for the Linear Array were significantly lower in FFPE samples compared to detection in exfoliated specimens (McNemar's p-value of 0.02 and 0.03 , and 0.01 , respectively).

\section{Discussion}

Very few studies have evaluated the performance of two or more methods in parallel in FFPE specimens [28-30]. In the present study, we evaluated simultaneously four different methods that not only varied in the set of primers used, but also in the sample processing and DNA extraction protocols of the independent laboratories. Even with such marked differences in the protocols, we observed good overall performance for all methods in the detection of HPV in FFPE specimens.

With the exception of the Onclarity assay, the genotyping performance of these HPV methods has been previously evaluated in FFPE specimens. Our findings are very much in agreement with other reports indicating a good to excellent performance of SPF-LiPA 25 , Inno-LiPA and the Linear Array method. Previous studies evaluating the SPF-LiPA 25 assay in FFPE and cytology 
Table 4 Human papillomavirus (HPV) genotyping agreement for paired cytology and tissue specimens ${ }^{\mathrm{a}}$ tested with different genotyping methods

\begin{tabular}{|c|c|c|c|c|c|c|c|c|c|c|c|c|}
\hline Overall agreement & \multicolumn{3}{|c|}{ Onclarity } & \multicolumn{3}{|c|}{ Inno-LiPA } & \multicolumn{3}{|c|}{ Linear Array } & \multicolumn{3}{|c|}{ SPF-LiPA 25} \\
\hline with cytology & \multicolumn{3}{|c|}{ N (\%) } & \multicolumn{3}{|c|}{ N (\%) } & \multicolumn{3}{|c|}{ N (\%) } & \multicolumn{3}{|c|}{ N (\%) } \\
\hline Identical & \multicolumn{3}{|c|}{$32(53.3)$} & \multicolumn{3}{|c|}{$34(56.7)$} & \multicolumn{3}{|c|}{$32(53.3)$} & \multicolumn{3}{|c|}{$37(61.7)$} \\
\hline Compatible & \multicolumn{3}{|c|}{$19(31.7)$} & \multicolumn{3}{|c|}{$17(28.3)$} & \multicolumn{3}{|c|}{$15(25.0)$} & \multicolumn{3}{|c|}{$20(33.3)$} \\
\hline Discrepant & \multicolumn{3}{|c|}{$9(15.0)$} & \multicolumn{3}{|c|}{$9(15.0)$} & \multicolumn{3}{|c|}{$13(21.7)$} & \multicolumn{3}{|c|}{$3(5.0)$} \\
\hline HPV type & $\%^{\mathrm{a}}$ & kappa & $p$ & $\%^{b}$ & kappa & $p$ & $\%^{a}$ & kappa & $p$ & $\%^{a}$ & kappa & $p$ \\
\hline Any oncogenic type ${ }^{c}$ & 90.0 & NA & & 85.0 & NA & & 80.0 & NA & & 95.0 & NA & \\
\hline \multicolumn{13}{|l|}{ Single probe } \\
\hline HPV 16 & 83.3 & 0.7 & 0.1 & 88.3 & 0.8 & 0.02 & 86.7 & 0.7 & 0.01 & 88.3 & 0.8 & 0.5 \\
\hline HPV 18 & 95 & 0.8 & 1.0 & 96.7 & 0.9 & 0.5 & 93.3 & 0.7 & 0.1 & 98.3 & 0.9 & 1.0 \\
\hline HPV 31 & 95 & 0.8 & 1.0 & 98.3 & 0.9 & 1.0 & 96.7 & 0.8 & 0.5 & 98.3 & 0.9 & 1.0 \\
\hline HPV 33 & NA & & & 100 & 1.0 & NA & 100 & 1.0 & NA & 100 & 1.0 & NA \\
\hline HPV 35 & NA & & & 98.3 & NA & & 98.3 & NA & & 98.3 & NA & \\
\hline HPV 39 & NA & & & 96.7 & 0.7 & 0.5 & 96.7 & 0.7 & 0.5 & 96.7 & 0.7 & 0.5 \\
\hline HPV 45 & 91.7 & 0.6 & 0.1 & 90.0 & 0.4 & 0.03 & 91.7 & 0.5 & 0.1 & 95.0 & 0.7 & 0.3 \\
\hline HPV 51 & 91.7 & 0.3 & 0.1 & 93.3 & 0.5 & 0.1 & 91.7 & 0.3 & 0.1 & 93.3 & 0.5 & 0.1 \\
\hline HPV $52^{d}$ & 98.3 & 0.9 & 1.0 & 95.0 & 0.6 & 0.3 & 93.3 & 0.5 & 0.1 & 96.7 & 0.8 & 1.0 \\
\hline HPV 56 & NA & & & 95.0 & 0.4 & 0.3 & 95.0 & 0.4 & 0.3 & 95.0 & 0.4 & 0.3 \\
\hline HPV 58 & NA & & & 96.7 & 0.7 & 0.5 & 96.7 & 0.7 & 0.5 & 96.7 & 0.7 & 0.5 \\
\hline HPV 59 & NA & & & $\mathrm{NA}$ & & & 100 & 1.0 & NA & 98.3 & 0.7 & 1.0 \\
\hline HPV 66 & NA & & & $\mathrm{NA}$ & & & NA & & & 95.0 & 0.4 & 0.3 \\
\hline HPV 68 & NA & & & NA & & & 98.3 & 0.7 & 1.0 & NA & & \\
\hline
\end{tabular}

${ }^{a}$ Genotyping agreement for 60 paired cytology- and formalin-fixed and paraffin embedded (FFPE) specimens in the SUCCEED study; Onclarity, The BD Onclarity ${ }^{\mathrm{T} M}$ HPV Assay; Inno-LiPA, The Inno-LiPA system; Linear Array, The PGMY09/11 Linear Array, and SPF-LiPA 25 , The SPF 10 -DEIA, LiPA 25 (version 1); Identical, same number and type identified; Compatible, at least one type in common identified; Discrepant, no type in common identified or FFPE sample HPV negative; ${ }^{a}$ Only single probes, but including probe combined probe 52/33/35/58 for Linear Array; ${ }^{\mathrm{b}}$ Percentage of overall agreement; ${ }^{\mathrm{C}}$ Includes a specimen positive for any of the $14 \mathrm{HPV}$ types; ${ }^{d}$ HPV 52 was positively identified in the linear Array test when the presence of HPV 33, 35, and 58 was excluded; $p$, p-value of the McNemar test; NA, not apply or cannot be calculated

specimens found an overall percentage agreements greater than $90 \%[13,15,31]$. The SPF-LiPA 25 has been also compared with other methods, such as the Genomica assay (primers MY09/1) [29] and the Linear Array assay [28], with similar results. Although there were methodological differences in those studies (genotyping method used for cytology specimens, grade of cervical lesion and time window between the testing of the cytological and tissue specimens), they all reported good agreement between $\mathrm{SPF}_{10}-\mathrm{LiPA}_{25}$ and the other tests. The SPF-LiPA 25 method uses the general primer set, designed as $\mathrm{SPF}_{10}$, which amplifies a fragment of only 65 bp of L1 region of the HPV genome [26]. This primer set has a high sensitivity for HPV detection, which makes it particularly suitable for assessing HPV in FFPE specimens.

Good performance for the Inno-LiPA method in FFPE versus cytology specimens has been also reported in previous studies. One Italian study compared the Inno-LiPA genotyping results from FFPE samples with the Linear Array results from the paired cytology specimens and reported an overall kappa value of 0.85 [14]. Similarly, a Slovenian study compared the performance of a real time PCR assay versus the Inno-LiPA method in 31 FFPE cervical cancer specimens, reporting $100 \%$ genotype agreement [32]. The Inno-LiPA method also performed well in FFPE specimens from other HPV-related cancers, such as head and neck [33] and vulva [30, 34].

To our knowledge, no previous study has compared FFPE samples tested by Linear Array with cytological results by Linear Array or any other method. We saw similar HPV genotyping results for FFPE and cytology specimens tested by Linear Array (agreement $80 \%$ ). As mentioned above, Linear Array has been directly compared with SPF-LiPA 25 and with Inno-LiPA in FFPE cervical [35] and vulvar specimens [30], respectively. Such studies showed good concordance between the methods for detecting any high-risk types. However, both previous studies [30, 35] and our study found that the sensitivity of Linear Array was lower compared to other methods. The lower sensitivity of Linear Array in FFPE samples could be attributed to DNA fragmentation; the 
Linear Array method targets an amplicon region much larger than other methods (450 bp), which can result in reduced amplification efficiency [36]. A limitation of Linear Array is the lack of an individual probe for HPV 52. Since HPV52 is detected in a mixed probe together with HPV-33, -35 , or -58 , multiple infections between HPV52 and one of these types cannot be detected.

Our study is the first one to test the Onclarity genotyping method using FFPE specimens. Previous studies have found that this method performs well in cytology specimens in comparison with other methods such as Hybrid Capture 2, Linear Array and Line Blot Assay [37]. Likewise a comprehensive study that compared seven genotyping methods in cytology specimens showed that Onclarity assay has a sensitivity of $95 \%$ for detection of CIN3+ [38]. Thus, the good performance of Onclarity in FFPE specimens is consistent with previous studies using cytology specimens. The BD Onclarity ${ }^{\text {Tm }}$ HPV Assay (BD Diagnostics) uses real-time PCR to detect 14 HR HPV types, six of which are tested individually while the remaining HPV types are grouped (33_58), (56_59_66) and (35_39_68). The HPV typing using multiple types in a single channel prevented the evaluation of the type specific agreement for these combined types and may limit the use of the assay when individual genotyping of these combined types is required.

We observed that independent of the genotyping method used, overall HPV prevalence (any type versus none) in the FFPE specimens (between 80 to $95 \%$ across methods) was lower than in cytology specimens (100\% with Linear Array), as has been found in some previous studies [7, 15, 31], but not others [13]. Although the sample size was too limited for a formal test, we did not see evidence of type-specific failures for any of the evaluated methods (Additional file 1: Table S1). We noted a difference in the detection of multiple types, with the Onclarity and SPF-LiPA 25 tests detecting more multiple types than either the Inno-LiPA or Linear Array tests. This may be related to smaller target sequences of these two assays, or to the previously described reduced performance of select L1 primer designs in detecting multiple infections [39]. While a smaller amplicon size could potentially have a higher risk of cross-sample contamination, we did not observe any sign of contamination in our study.

DNA fragmentation and DNA-protein cross-linking by formaldehyde exposure, as well as the presence of paraffin, can impact both DNA yield and PCR amplification efficiency. Our study evaluated combinations of DNA extraction methods and HPV genotyping assays performed in different laboratories. Therefore, the differences observed in this study cannot be directly attributed to assay performance versus the DNA extraction approach used. However, generally, the differences between results from different laboratories were minor and seemed to be related mostly to amplicon size.

Strengths of the current analysis are the complete histological characterization of the selected specimens, and the short interval between cytological and histological specimen collection. A limitation of our study was the lack of parallel genotyping data from cytology specimens for all the tested genotyping methods.

\section{Conclusions}

HPV genotyping from FFPE tissues showed high intraand inter-laboratory reproducibility in our study; the use of any of the tested HPV genotyping methods in expert laboratories can provide reliable identification of the most important high risk HPV types in FFPE specimens. However, there remains concern that reliability of tissuebased genotyping may be worse in less experienced and less rigorous laboratories, as indicated by a WHO HPV laboratory comparison study including 29 centers across the world, which reported lower proficiency measures for HPV detection despite the use of standardized DNA samples [40], rather than whole tissue sections as in the current study. Thus, it can be expected that there is higher variation of proficiency for tissue-based genotyping in less experienced and less rigorous laboratories.

\section{Additional files}

Additional file 1: Table S1. Human papillomavirus (HPV) genotyping results for 60 formalin-fixed and paraffin embedded (FFPE) specimens tested in four different laboratories compared to the paired cytology specimen tested by Linear Array. (DOC $101 \mathrm{~kb}$ )

Additional file 2: Table S2. Human papillomavirus (HPV) genotyping reproducibility for 6 formalin-fixed and paraffin embedded (FFPE) specimens by genotyping method and the paired HPV cytological result in the SUCCEED study. (DOC $34 \mathrm{~kb}$ )

Additional file 3: Table S3. Human papillomavirus (HPV) genotyping agreement for tissue specimens* tested with different genotyping methods. (DOC $31 \mathrm{~kb})$

\section{Abbreviations}

HPV: Human papillomavirus; FFPE: Formalin-fixed, paraffin-embedded; PCR: Polymerase chain reaction; CIN: Cervical intraepithelial neoplasia; LEEP: Loop electrosurgical excision procedure;

EDTA: Ethylenediaminetetraacetic acid; PK: Proteinase K; DEIA: DNA enzyme immunoassay; WHO: World Health Organization.

\section{Competing interests}

Becton Dickinson Diagnostics conducted testing of the specimens without charge. Laurence Vaughan is an employee of Becton Dickinson Diagnostics Sparks, MD, US. Wim Quint, Leen-Jan van Doorn and Bernhard Kleter are employed by DDL Diagnostic Laboratory, Rijswijk, The Netherlands. The other authors declare that they have no conflicts of interest.

\section{Authors' contributions}

NW, JK, ACH, AH, MS- contributed to conception and the study design, MS, NW, SDT, SW, JW, RZ provided cytological and FFPE specimens from SUCCEED, WQ, LVD, BK, LV, MG, CW, coordinated and performed laboratory methods, FAC, NW, JK, analysed and interpreted all the data, FAC, NW, JK wrote a first draft of the paper. All authors read, commented on and approved the final manuscript. 


\section{Acknowledgments}

We thank Patricia Agreda and Ryan Schwab from Becton, Dickinson and Company for HPV genotyping with The BD Onclarity ${ }^{T M}$ HPV Assay.

\section{Funding}

Intramural Research Program of the NCI (Z01 CP010124: Cervical cancer and human papillomavirus (HPV) infection).

\section{Disclosures}

The BD Onclarity ${ }^{\mathrm{TM}}$ HPV Assay is currently not available for sale or use in the United States.

\section{Author details}

${ }^{1}$ Division of Cancer Epidemiology and Genetics, National Cancer Institute, Bethesda, MD, USA. ²DL, Diagnostic Laboratory, Rijswijk, The Netherlands. ${ }^{3}$ Viral Oncology Program, The Ohio State University Comprehensive Cancer Center, Columbus, OH, USA. ${ }^{4}$ Department of Pathology, School of Medicine, University of New Mexico, Albuquerque, NM, USA. ${ }^{5}$ BD Diagnostics, Sparks, MD, USA. 'Division of Cancer Etiology, Department of Population Sciences, Beckman Research Institute and the City of Hope, Duarte, CA, USA. ${ }^{7}$ Departments of Pathology and Obstetrics and Gynecology, University of Oklahoma Health Sciences Center, Oklahoma City, OK, USA. ${ }^{8}$ Division of Cancer Epidemiology \& Genetics, NCI Shady Grove, 9609 Medical Center Drive 7E114, Rockville, MD 20850, USA.

Received: 3 July 2015 Accepted: 17 November 2015

\section{Published online: 25 November 2015}

\section{References}

1. Walboomers JM, Jacobs MV, Manos MM, Bosch FX, Kummer JA, Shah KV et al. Human papillomavirus is a necessary cause of invasive cervical cancer worldwide. J Pathol. 1999;189(1):12-9.

2. Munoz N, Bosch FX, de Sanjose S, Herrero R, Castellsague X, Shah KV, et al. Epidemiologic classification of human papillomavirus types associated with cervical cancer. N Engl J Med. 2003;348(6):518-27.

3. Luhn P, Wentzensen N. HPV-based tests for cervical cancer screening and management of cervical disease. Curr Obstet Gynecol Rep. 2013;2(2):76-85.

4. Wheeler CM, Hunt WC, Joste NE, Key CR, Quint WG, Castle PE. Human papillomavirus genotype distributions: implications for vaccination and cancer screening in the United States. J Natl Cancer Inst. 2009;101(7):475-87.

5. Ho GY, Burk RD, Klein S, Kadish AS, Chang CJ, Palan P, et al. Persistent genital human papillomavirus infection as a risk factor for persistent cervical dysplasia. J Natl Cancer Inst. 1995;87(18):1365-71.

6. Quint W, Jenkins D, Molijn A, Struijk L, van de Sandt M, Doorbar J, et al. One virus, one lesion-individual components of CIN lesions contain a specific HPV type. J Pathol. 2012;227(1):62-71.

7. van der Marel J, Quint WG, Schiffman M, van de Sandt MM, Zuna RE, Dunn ST, et al. Molecular mapping of high-grade cervical intraepithelial neoplasia shows etiological dominance of HPV16. Int J Cancer. 2012;131(6):E946-53.

8. Schiffman M, Wentzensen N, Wacholder S, Kinney W, Gage JC, Castle PE. Human papillomavirus testing in the prevention of cervical cancer. J Nat Cancer Inst. 2011;103(5):368-83.

9. Greer CE, Peterson SL, Kiviat NB, Manos MM. PCR amplification from paraffin-embedded tissues. Effects of fixative and fixation time. Am J Clin Pathol. 1991:95(2):117-24.

10. Greer CE, Lund JK, Manos MM. PCR amplification from paraffin-embedded tissues: recommendations on fixatives for long-term storage and prospective studies. PCR Methods Appl. 1991;1(1):46-50.

11. Greer CE, Wheeler CM, Manos MM. Sample preparation and PCR amplification from paraffin-embedded tissues. PCR Methods Appl. 1994;3(6): S113-22.

12. Baay MF, Quint WG, Koudstaal J, Hollema H, Duk JM, Burger MP, et al. Comprehensive study of several general and type-specific primer pairs for detection of human papillomavirus DNA by PCR in paraffin-embedded cervical carcinomas. J Clin Microbiol. 1996;34(3):745-7.

13. Dal Bello B, Spinillo A, Alberizzi P, Cesari S, Gardella B, Silini EM. Validation of the SPF10 LiPA human papillomavirus typing assay using formalin-fixed paraffin-embedded cervical biopsy samples. J Clin Microbiol. 2009;47(7): 2175-80.

14. Dona MG, Ronchetti L, Giuliani M, Carosi M, Rollo F, Congiu M, et al. Performance of the linear array HPV genotyping test on paired cytological and formalin-fixed, paraffin-embedded cervical samples. J Mol Diagn. 2013; 15(3):373-9.

15. Gravitt PE, van Doorn $\sqcup$, Quint W, Schiffman M, Hildesheim A, Glass AG, et al. Human papillomavirus (HPV) genotyping using paired exfoliated cervicovaginal cells and paraffin-embedded tissues to highlight difficulties in attributing HPV types to specific lesions. J Clin Microbiol. 2007;45(10):3245-50.

16. Agreda PM, Beitman GH, Gutierrez EC, Harris JM, Koch KR, LaViers WD, et al. Long-term stability of human genomic and human papillomavirus DNA stored in BD SurePath and Hologic PreservCyt liquid-based cytology media. J Clin Microbiol. 2013;51(8):2702-6.

17. Duval K, Aubin RA, Elliott J, Gorn-Hondermann I, Birnboim HC, Jonker D, et al. Optimized manual and automated recovery of amplifiable DNA from tissues preserved in buffered formalin and alcohol-based fixative. Forensic Sci Int Genet. 2010;4(2):80-8.

18. Kotorashvili A, Ramnauth A, Liu C, Lin J, Ye K, Kim R, et al. Effective DNA/ RNA co-extraction for analysis of microRNAs, mRNAs, and genomic DNA from formalin-fixed paraffin-embedded specimens. PLoS ONE. 2012;7(4):e34683.

19. Wang SS, Zuna RE, Wentzensen N, Dunn ST, Sherman ME, Gold MA, et al. Human papillomavirus cofactors by disease progression and human papillomavirus types in the study to understand cervical cancer early endpoints and determinants. Cancer Epidemiol Biomarkers Prev. 2009;18(1): $113-20$

20. Wentzensen N, Schiffman M, Dunn T, Zuna RE, Gold MA, Allen RA, et al. Multiple human papillomavirus genotype infections in cervical cancer progression in the study to understand cervical cancer early endpoints and determinants. Int J Cancer. 2009;125(9):2151-8.

21. Koshiol J, Rotunno M, Gillison ML, Van Doorn LJ, Chaturvedi AK, Tarantini L, et al. Assessment of human papillomavirus in lung tumor tissue. J Natl Cancer Inst. 2011;103(6):501-7.

22. Gravitt PE, Peyton CL, Alessi TQ, Wheeler CM, Coutlee F, Hildesheim A, et al. Improved amplification of genital human papillomaviruses. J Clin Microbiol. 2000;38(1):357-61.

23. Peyton CL, Gravitt PE, Hunt WC, Hundley RS, Zhao M, Apple RJ, et al. Determinants of genital human papillomavirus detection in a US population. J Infect Dis. 2001;183(11):1554-64.

24. Wheeler CM, Hunt WC, Cuzick J, Langsfeld E, Pearse A, Montoya GD, et al. A population-based study of human papillomavirus genotype prevalence in the United States: baseline measures prior to mass human papillomavirus vaccination. Int J Cancer. 2013;132(1):198-207.

25. Kleter B, van Doorn LJ, Schrauwen L, Molijn A, Sastrowijoto S, ter Schegget J, et al. Development and clinical evaluation of a highly sensitive PCRreverse hybridization line probe assay for detection and identification of anogenital human papillomavirus. J Clin Microbiol. 1999;37(8):2508-17.

26. Kleter B, van Doorn LJ, ter Schegget J, Schrauwen L, van Krimpen K, Burger $M$, et al. Novel short-fragment PCR assay for highly sensitive broadspectrum detection of anogenital human papillomaviruses. Am J Pathol. 1998;153(6):1731-9.

27. van Doorn LJ, Molijn A, Kleter B, Quint W, Colau B. Highly effective detection of human papillomavirus 16 and 18 DNA by a testing algorithm combining broad-spectrum and type-specific PCR. J Clin Microbiol. 2006; 44(9):3292-8.

28. Hariri S, Steinau M, Rinas A, Gargano JW, Ludema C, Unger ER, et al. HPV genotypes in high grade cervical lesions and invasive cervical carcinoma as detected by two commercial DNA assays, North Carolina, 2001-2006. PLoS ONE. 2012;7(3):e34044

29. Perez C, Klaustermeier JE, Alemany L, Tous S, de Sanjose S, Velasco J. Comparison of 2 different PCR-based technologies for the detection of human papilloma virus from paraffin-embedded tissue: genomica clinical arrays versus SPF(10)-LiPA(25). Diagn Mol Pathol. 2012;21(1):45-52.

30. Tan SE, Garland SM, Rumbold AR, Tabrizi SN. Human papillomavirus genotyping using archival vulval dysplastic or neoplastic biopsy tissues: comparison between the INNO-LiPA and linear array assays. J Clin Microbiol. 2010;48(4):1458-60.

31. Quint WG, Scholte G, van Doorn LJ, Kleter B, Smits PH, Lindeman J. Comparative analysis of human papillomavirus infections in cervical scrapes and biopsy specimens by general SPF(10) PCR and HPV genotyping. J Pathol. 2001;194(1):51-8.

32. Kocjan BJ, Seme K, Poljak M. Comparison of the Abbott RealTime High Risk HPV test and INNO-LiPA HPV Genotyping Extra test for the detection of human papillomaviruses in formalin-fixed, paraffin-embedded cervical cancer specimens. J Virol Methods. 2011;175(1):117-9. 
33. Kocjan BJ, Maver PJ, Hosnjak L, Zidar N, Odar K, Gale N, et al. Comparative evaluation of the Abbott RealTime High Risk HPV test and INNO-LiPA HPV Genotyping Extra test for detecting and identifying human papillomaviruses in archival tissue specimens of head and neck cancers. Acta Dermatovenerol Alp Panonica Adriat. 2012;21(4):73-5.

34. Tan SE, Garland SM, Rumbold AR, Tabrizi SN. Comparison of the INNO-LiPA and PapType assays for detection of human papillomavirus in archival vulva dysplasia and/or neoplasia tissue biopsy specimens. J Clin Microbiol. 2011; 49(11):3980-2.

35. Hariri S, Unger ER, Sternberg M, Dunne EF, Swan D, Patel S, et al. Prevalence of genital human papillomavirus among females in the United States, the National Health And Nutrition Examination Survey, 2003-2006. J Infect Dis. 2011:204(4):566-73.

36. Ferrer I, Armstrong J, Capellari S, Parchi P, Arzberger T, Bell J, et al. Effects of formalin fixation, paraffin embedding, and time of storage on DNA preservation in brain tissue: a BrainNet Europe study. Brain Pathol. 2007; 17(3):297-303.

37. Castle PE, Gutierrez EC, Leitch SV, Maus CE, McMillian RA, Nussbaumer WA, et al. Evaluation of a new DNA test for detection of carcinogenic human papillomavirus. J Clin Microbiol. 2011;49(8):3029-32.

38. Szarewski A, Mesher D, Cadman L, Austin J, Ashdown-Barr L, Ho L, et al. Comparison of seven tests for high-grade cervical intraepithelial neoplasia in women with abnormal smears: the Predictors 2 study. J Clin Microbiol. 2012;50(6):1867-73.

39. Mori S, Nakao S, Kukimoto I, Kusumoto-Matsuo R, Kondo K, Kanda T. Biased amplification of human papillomavirus DNA in specimens containing multiple human papillomavirus types by PCR with consensus primers. Cancer Sci. 2011;102(6):1223-7.

40. Eklund C, Zhou T, Dillner J, Network ftWHPL. Global Proficiency Study of Human Papillomavirus Genotyping. J Clin Microbiol. 2010;48(11):4147-55.

\section{Submit your next manuscript to BioMed Central and we will help you at every step:}

- We accept pre-submission inquiries

- Our selector tool helps you to find the most relevant journal

- We provide round the clock customer support

- Convenient online submission

- Thorough peer review

- Inclusion in PubMed and all major indexing services

- Maximum visibility for your research 